\title{
Bereitstellung von Dendromasse für die Versorgung von Biomassekraftwerken - Analyse am Beispiel des Standorts Elsterwerda
}

\author{
Paul Fiedler, Mareike Schultze, Herbert Sonntag
}

\section{Einleitung}

\section{Hintergrund}

Energieversorgung steht im Spannungsverhältnis zwischen Versorgungssicherheit, Wirtschaftlichkeit, Umweltverträglichkeit und der Notwendigkeit gesellschaftlicher Akzeptanz.

Regenerative Energien sind geeignet, die sich perspektivisch öffnende Lücke zwischen der weltweit steigenden Energienachfrage und der unsicheren Expansion des Energieangebots zumindest teilweise zu schließen (BMWI, BMU, 2006). Neben ihrem Beitrag zur Ressourcenschonung und zum Klimaschutz sind sie ein wichtiger Wirtschaftsfaktor (BMU, 2006) und verringern die Abhängigkeit von Energieimporten.

Unter den regenerativen Energien zeichnen sich die Bio-Energien auf der Basis nachwachsender Rohstoffe durch ihr großes Potential und die vielfältigen Einsatzmöglichkeiten aus. Sie können für die Erzeugung von Elektrizität, für die Gewinnung von Wärme und für die Produktion von Kraftstoffen genutzt werden. Zudem sind sie kontinuierlich verfügbar und lagerfähig. Langfristig könnte die Nutzung von Biomasse etwa $10 \%$ zur Energiebereitstellung in Deutschland beitragen (vgl. BMU, 2005).

Die Voraussetzungen für die Nutzung von Bio-Energien sind sehr günstig. Ihre Umweltverträglichkeit steht außerhalb jeder Diskussion und die gesellschaftliche Akzeptanz ist grundsätzlich hoch. Insbesondere in strukturschwachen Regionen kann die Nutzung eigener Biomasseressourcen einen wichtigen Beitrag zur regionalen Wertschöpfung leisten. Große Wachstumschancen im Bereich der Biomasse werden der Verwendung bisher ungenutzter Holzpotentiale zugesprochen (Auer, 2005).

Die Logistik spielt hinsichtlich der Wirtschaftlichkeit der Nutzung von Dendromasse (Energieholz) eine wichtige Rolle. Die Kosten der Bereitstellung übersteigen häufig die des eigentlichen Materialwertes. Umso wichtiger erscheint es, effiziente Bereitstellungskonzepte zu entwickeln.

\section{Projektaufgabe}

Die Projektaufgabe bestand darin die Umstellung der Versorgung des Biomassekraftwerkes Elsterwerda von Altholz auf Waldrestholz nach finanziellen Gesichtspunkten zu prüfen. Hintergrund ist die derzeitige Entwicklung auf dem Altholzmarkt. Stellte
Altholz bisher eine reichlich verfügbare und kostengünstige Rohstoffquelle dar, so sind die Mengen zunehmend abgeschöpft. Allgemein wird mit einer Verknappung und mit ansteigenden Preisen gerechnet (Weimar und Mantau, 2005). Zusätzlich werden die Transportentfernungen und damit die Bereitstellungskosten für Altholz steigen. Waldrestholz hingegen steht flächendeckend zur Verfügung und wird nur unzureichend genutzt.

Besonderen Wert bei der Umsetzung der Aufgabe, wurde auf den Ursprung der Dendromasse gelegt. Diese sollte ausschließlich aus regionalen Ressourcen stammen, in diesem Untersuchungsfall dem Landkreis Elbe-Elster/Brandenburg. Neben der Ermittlung geeigneter Dendromasseprodukte stand die Untersuchung der Logistik- und Wertschöpfungskette im Mittelpunkt. Für das Kraftwerk Elsterwerda sollten die Kosten für die Versorgung mit Waldrestholz anhand einer beispielhaften Wertschöpfungskette ermittelt und mit den derzeitigen Kosten verglichen werden. Auf Basis der Ergebnisse sollte ein Handlungsvorschlag gemacht bzw. Alternativen zur bisherigen ausschließlichen Altholznutzung aufgezeigt werden.

\section{Grundlagen und Vorgehensweise}

Unter dem Begriff „Dendromasse“ wird holzartige Biomasse verstanden, die einer energetischen Nutzung zugeführt wird. Dies betrifft sowohl Holzsortimente, die direkt durch thermische Aufarbeitung genutzt werden, als auch solche die in einem ersten Schritt in ein hochverdichtetes Zwischenprodukt gewandelt und erst anschließend in einem zweiten Aufbereitungsschritt einer energetischen Nutzung zugeführt werden. In Abb. 1 sind unterschiedliche Bereitstellungspfade für Dendromasse dargestellt.

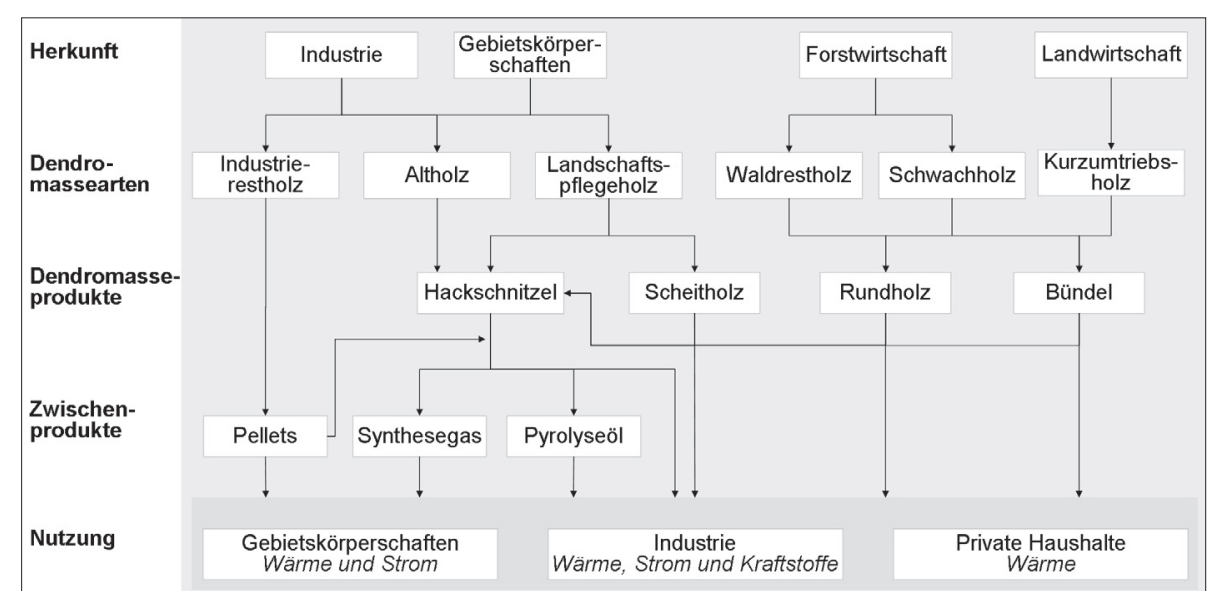

Abb. 1: Bereitstellungspfade Dendromasse, Quelle: nach Kaltschmitt und Hartmann (2001) 
Wie aus der Abb. 1 zu ersehen ist, kann frische Dendromasse in der Forstwirtschaft, der Landwirtschaft und bei der Landschaftspflege anfallen. Potentialmodelle zu Kurzumtriebsplantagen auf landwirtschaftlichen Flächen und zu Biomasse aus der Landschaftspflege sind derzeit noch nicht verfügbar. Obwohl diesen Dendromassearten ein hohes Potential zugesprochen wird, beschränkt sich daher diese Analyse auf Waldrestholz und Schwachholz aus der Forstwirtschaft. Waldrestholz fällt als Nebenprodukt bei der Ernte von Holz für die stoffliche Nutzung an. Holz unterhalb der Nutzungsgrenze und Kronenmaterial verbleiben zurzeit überwiegend ungenutzt im Wald. Schwachholz ist Holz aus der Jungbestandspflege, dessen Durchmesser zu gering für eine stoffliche Nutzung ist. Unter Beachtung der Grundsätze für eine nachhaltige Nutzung steht ein Teil dieses Materials für die Energieerzeugung zur Verfügung.

An der Wertschöpfungskette Dendromasse sind eine Reihe von Akteuren beteiligt. Auf der Angebotsseite sind das die Forstverwaltung, private Waldbesitzer und Forstdienstleister.

Die Abnehmerseite ist sehr differenziert. Es handelt sich hier um Betreiber von Konversionsanlagen in den unterschiedlichsten Dimensionierungen. Privatpersonen kommen als Abnehmer kleinerer Mengen ebenfalls in Betracht. Eine wichtige Rolle im Bereitstellungsprozess spielen die Dienstleister. Sie können sowohl die Prozessschritte Ernte und Aufbereitung als auch Transport und Lagerung übernehmen. Die Vielzahl der Ablaufprozesse bietet hier auch eine ebenso große Zahl von Varianten der Arbeitsteilung.

$\underset{\text { Waldbesitzer/Forstwirt/Forstdienstleister }}{\longrightarrow \text { Materialfluss }}$

Abb. 2: Wertschöpfungskette forstlicher Dendromasse

Je nach den Anforderungen an die Dendromassequalität sind unterschiedliche Verfahrensschritte notwendig. Entscheidend für die Gestaltung der Bereitstellungskette ist die Herstellung eines transportfähigen Dendromasseprodukts. Waldrestholz und Schwachholz werden häufig direkt am Ort des Anfalls zu Waldhackschnitzeln zerkleinert. Alternativ ist die Aufbereitung zu Bündeln oder Rundholzabschnitten möglich. Privathaushalte nehmen häufig Scheitholz ab.

Die Bereitstellungskosten zur Versorgung einer Anlage mit Dendromasse hängen ab von:

- den Anforderungen an Menge und Qualität der Dendromasse, die sich aus Art und Dimensionierung der Konversionsanlage ergeben,

- den verfügbaren Dendromasseressourcen,

- der Herkunft der Dendromasse und

- den eingesetzten Verfahren für Ernte, Aufbereitung, Lagerung, Umschlag und Transport.

Die Vorgehensweise zur Erstellung eines Versorgungskonzepts für das Biomassekraftwerk Elsterwerda ist in Abb. 3 dargestellt. Zunächst werden die Anforderungen an die Bereitstellung definiert. Es folgt eine Untersuchung des Landkreises Elbe-Elster hinsichtlich seiner Eignung für die Versorgung des Biomasseheizkraftwerkes Elsterwerda. Wichtige Aspekte sind die infrastrukturelle Anbindung des Standorts und die räumliche Verteilung nachhaltig vorhandener Dendromassepotentiale. Darauf aufbauend werden exemplarische Bereitstellungsketten zur Versorgung des Standorts definiert und monetär bewertet. Die Bewertung des Versorgungskonzepts und das Aufzeigen von Handlungsalternativen erfolgt entsprechend der eingangs formulierten qualitativen, quantitativen und monetären Vorgaben.

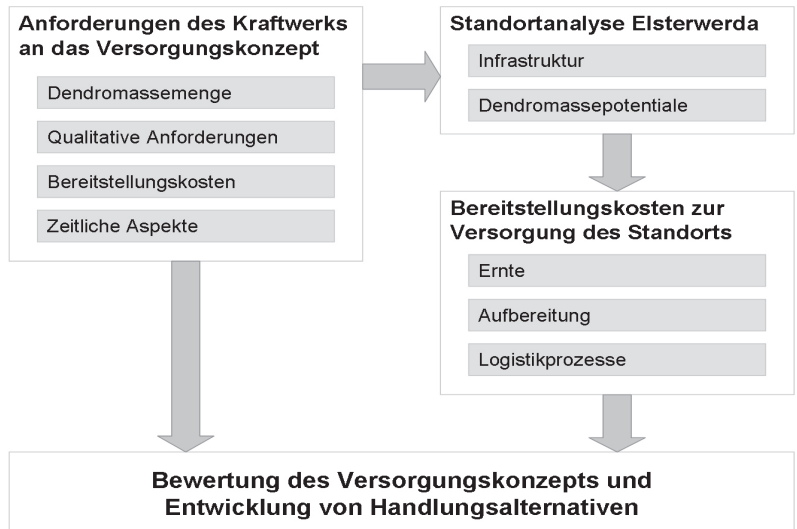

Abb. 3: Vorgehensweise zur Erstellung und Bewertung des Versorgungskonzepts

\section{Anforderungen an ein Konzept zur Ver- sorgung des Kraftwerks Elsterwerda}

Ein positives Betriebsergebnis zu erzielen, ist die Grundvoraussetzung für das wirtschaftliche Bestehen eines Biomasseheizkraftwerks. Im Fall der vorliegenden Untersuchung soll für das Biomassekraftwerk Elsterwerda geprüft werden, ob ein kostendeckender Betrieb auf der Basis von Waldrestholz möglich ist. Die Bereitstellungskosten dürfen einen definierten Anteil an den Einnahmen, d.h. an der Gesamtvergütung für Strom und Wärme, nicht überschreiten.

Das Kraftwerk Elsterwerda produziert Strom und Wärme bei einer Arbeitsleistung von 8.000 Volllaststunden pro Jahr. Der Schwerpunkt liegt mit 96.000 MWh/a auf der Stromproduktion. Weitere $24.000 \mathrm{MWh} / \mathrm{a}$ Wärme werden ins Fernwärmenetz der Stadt Elsterwerda eingespeist. Der vom Kraftwerk Elsterwerda bereitgestellte Strom wird nach den im Erneuerbare-Energien-Gesetz (EEG) festgelegten Sätzen vergütet. Die Bewertung der produzierten Wärmemenge erfolgte nach dem durchschnittlichen Heizölpreis 2005 (Tecson, 2006).

\begin{tabular}{|l|l|l|}
\hline Leistung & $\mathrm{MWe}_{\mathrm{I}}$ & 12,6 \\
\hline Stromproduktion & $\mathrm{MWh} / \mathrm{a}$ & 96.000 \\
\hline Wärmeproduktion & $\mathrm{MWh} / \mathrm{a}$ & 24.000 \\
\hline Holzbedarf & t atro/a & 96.000 \\
\hline Stromvergütung & $€ / \mathrm{a}$ & $8,3 \mathrm{Mio}$. \\
\hline Wert der produzierten Wärme & $€ / \mathrm{a}$ & $1,3 \mathrm{Mio}$. \\
\hline
\end{tabular}

Tab. 1 Kenndaten des Biomassekraftwerks Elsterwerda

Die Anlage hat einen jährlichen Brennstoffbedarf von $96.000 \mathrm{t}$ Trockenmasse ( $\mathrm{t}$ atro). Bisher wird diese Menge fast ausschließlich durch Altholz abgedeckt. Nach der 
geplanten Jahresproduktion am Standort Elsterwerda ergibt sich eine Gesamtvergütung für die bereitgestellte Strom- und Wärmemenge von etwa 9,5 Mio. €. Bei einer Kostenstruktur vergleichbar mit ähnlichen Anlagen liegt das Budget für die Bereitstellungskosten von Dendromasse bei $30-35 \%$ der Gesamtkosten.

Der Einkauf des Altholzes erfolgt frei Werk, wobei die Anlieferung ausschließlich per Lkw realisiert wird. Vor Ort gibt es keine Hackanlage, so dass ausschließlich Hackschnitzel eingekauft werden. Diese können eine Kantenlänge von bis zu $300 \mathrm{~mm}$ aufweisen. Der Preis für Altholz schwankt zwischen $10-40 € / t$ atro (Leible et al, 2003).

Die Anlage ist für das Verfeuern von Altholz aller Kategorien ausgelegt, das mit Störstoffen und chemischen Substanzen, wie z. B. Teer, Holzschutzmittel oder Chrom, belastet ist. Durch die notwendigen Reinigungs- und Filteranlagen liegen im Vergleich zur Verbrennung unbelasteter Holzsortimente die jährlichen Fixkosten deutlich höher.

Die Zufeuerung von Waldhackschnitzeln ist grundsätzlich möglich aber mit technischen Schwierigkeiten verbunden. Grund ist der hohe Wassergehalt (50 - $60 \%)$ von frischen Waldhackschnitzeln im Vergleich zu Altholz. Bei einem Wassergehalt von über $20 \%$ ist bei dem vorhandenen Kesselvolumen eine Drosselung der Anlagenleistung erforderlich. Um den kritischen Wassergehalt nicht zu überschreiten, ist eine konstante Zumischung einer definierten Menge Waldhackschnitzel zum Altholz erforderlich.

Die in Tabelle 2 dargestellten Anforderungen des Kraftwerks Elsterwerda dienen als Zielvorgabe für die Entwicklung des Versorgungskonzepts.

\begin{tabular}{|l|l|}
\hline Mengenbedarf & 96.000 t atro/a \\
\hline Dendromasseart & - Waldrestholz, Schwachholz \\
\cline { 2 - 2 } & - Altholz \\
\hline Mischung & $\begin{array}{l}\text { Maximaler Anteil Waldrestholz und } \\
\text { Schwachholz }\end{array}$ \\
\hline Dendromasseprodukt & Hackschnitzel \\
\hline $\begin{array}{l}\text { Qualtitative } \\
\text { Anforderungen }\end{array}$ & Kantenlänge < 300 mm \\
\cline { 2 - 2 } $\begin{array}{l}\text { Herkunft } \\
\text { Wassergehalt < 20\% }\end{array}$ \\
\hline Ort der Abnahme & Waldrestholz, Schwachholz: LK Elbe-Elster \\
\cline { 2 - 2 } $\begin{array}{l}\text { Zeitpunkt der } \\
\text { Bereitstellung }\end{array}$ & Eltholz: überregional \\
\hline $\begin{array}{l}\text { Methodik der } \\
\text { Bereitstellung }\end{array}$ & Bedarfsabhängig, möglichst kontinuierlich \\
\hline Budget & Anlieferung per LKW \\
\hline
\end{tabular}

Tab. 2: Zielvorgaben für die Versorgung des Kraftwerks Elsterwerda mit Waldhackschnitzeln

\section{Standortanalyse Elbe-Elster}

\section{Infrastruktur}

Für die Versorgung von Biomassekraftwerken mit Dendromasse stehen grundsätzlich die Verkehrsträger Straße, Schiene und Wasserstraße zur Verfügung. In diesem Abschnitt werden sie hinsichtlich ihrer Zweckmäßigkeit für die Versorgung des Standorts Elsterwerda untersucht.
Neben den vorhandenen Binnenhäfen werden hierbei die Holzverladebahnhöfe sowie die Straßenanbindungen betrachtet.

Die Einbindung von Bahn und Binnenschiff in die Versorgungskette erfordert einen straßengebundenen Vorlauf vom Ort der Biomasseproduktion zum Verladebahnhof bzw. Binnenhafen. Ein bedeutender Kostenfaktor ist der Umschlag der Güter von einem Verkehrsträger zum anderen. Für einen kostengünstigen Transport ist es daher ideal, wenn das Kraftwerk über einen Gleisanschluss verfügt bzw. direkt an einer Wasserstraße liegt, und damit der straßengebundene Nachlauf wegfällt.

Der einzige Holzverladebahnhof im Landkreis liegt in Finsterwalde und damit rund $30 \mathrm{~km}$ vom Standort Elsterwerda entfernt. Die nächstgelegenen Binnenhäfen sind 45 bzw. $30 \mathrm{~km}$ entfernt in Torgau und Riesa, Sachsen, angesiedelt. Für die Versorgung des Standorts Elsterwerda - gemäß der Zielvorgabe - aus regionalen Ressourcen kommt daher nur die Straße in Frage. In den weiteren Betrachtungen und bei der Ermittlung der Transportkosten wird daher ausschließlich der Verkehrsträger Straße berücksichtigt.

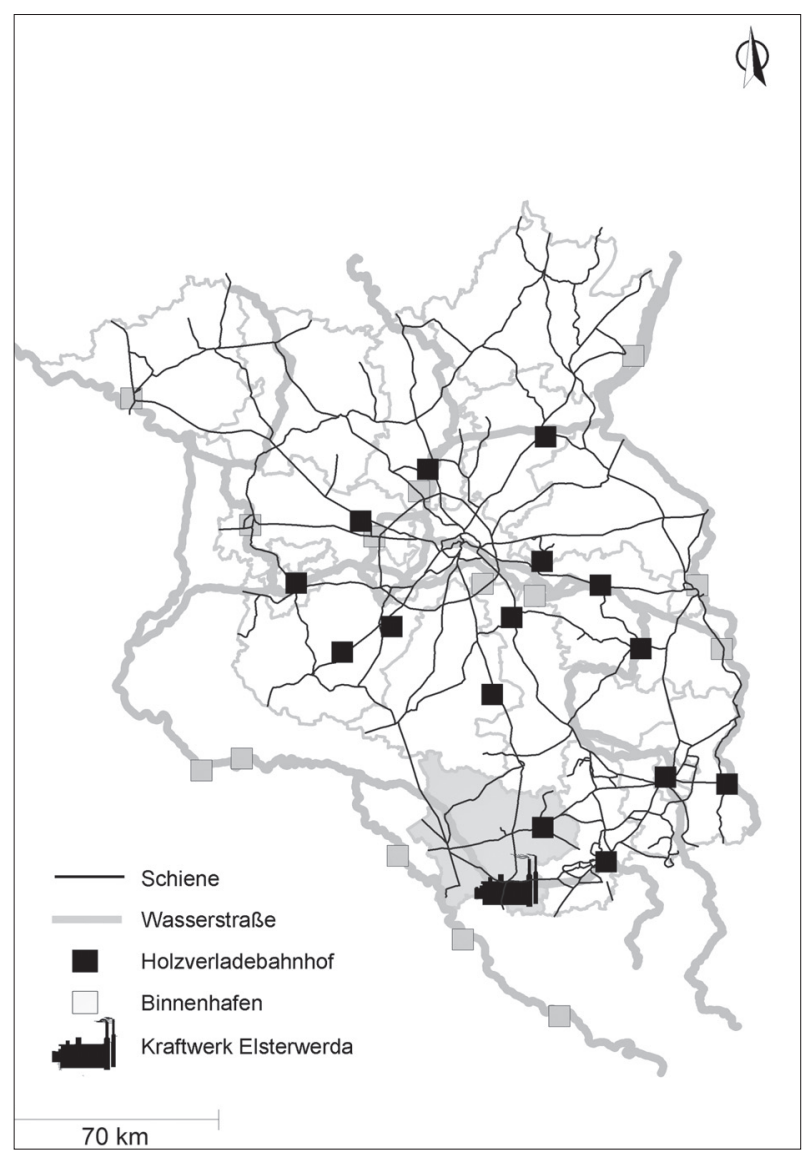

Abb. 4: Infrastrukturelle Anbindungen des Standorts Elsterwerda im Land Brandenburg

\section{Dendromassepotentiale}

Bei der Analyse der Dendromassepotentiale spielt neben den absoluten Vorkommen im Landkreis Elbe-Elster auch die räumliche Verteilung eine wichtige Rolle. Diese wurde mit Hilfe der GIS(Geographisches Informationssystem)Software Regiograph dargestellt. Diese Daten bilden die Grundlage, um die vorhandenen Holzpotentiale und das zu untersuchende Biomasseheizkraftwerk Elsterwerda in 
räumliche Relation zu setzen. Für die Berechnung der Transportkosten sind diese Daten elementar.

Die im Rahmen der Projektarbeit verwendeten Potentialdaten basieren auf Untersuchungen der Hamburger Bundesforschungsanstalt für Holz- und Forstwirtschaft (Dieter und Englert, 2001). Zur Potentialermittlung für den Landkreis Elbe-Elster wurden die Waldflächen in Brandenburg und Elbe-Elster (vgl. Tabelle 2) herangezogen. Die ermittelten Werte wurden mithilfe von regionalen Potentialwerten (Bilke, 2005) kalibriert. Die in Tabelle 3 dargestellten Daten liegen am unteren Ende der vorliegenden Abschätzungen und sind daher als Minimalwerte zu betrachten.

\begin{tabular}{|l|l|l|l|}
\hline & Deutschland & $\begin{array}{l}\text { Branden- } \\
\text { burg }^{\text {a) }}\end{array}$ & $\begin{array}{l}\text { Elbe- } \\
\text { Elster }\end{array}$ \\
\hline Waldfläche [ha] & 10,6 Mio. & 1 Mio. & 60.000 \\
\hline $\begin{array}{l}\text { Dendromassepotential } \\
\text { Gesamt [t atro/a] }\end{array}$ & 16,6 Mio. & 1.6 Mio. & 94.000 \\
\hline davon Schwachholz ${ }^{\text {b) }}[\mathrm{t}$ atro/a] & 7 Mio. & 0,7 Mio. & 40.000 \\
\hline davon Kronenholz [t atro/ha] & 9,6 Mio. & 0,9 Mio. & 55.000 \\
\hline $\begin{array}{l}\text { a) Abschätzung auf Basis der Waldfläche } \\
\text { b) Angaben bei einer unteren Aufarbeitungsgrenze von } 8 \mathrm{~cm}\end{array}$ \\
\hline
\end{tabular}

Tab. 3: Dendromassepotential aus der Forstwirtschaft in Deutschland, Brandenburg und im Landkreis Elbe Elster, Quellen: Dieter und Englert (2001), LSDB (2003), eigene Berechnungen

Nach dieser Schätzung ist im Landkreis Elbe-Elster mit einem Dendromassepotential von etwa $94.000 \mathrm{t}$ atro/a zu rechnen, die sich wie folgt um den Standort Elsterwerda verteilen (siehe Abb. 5).

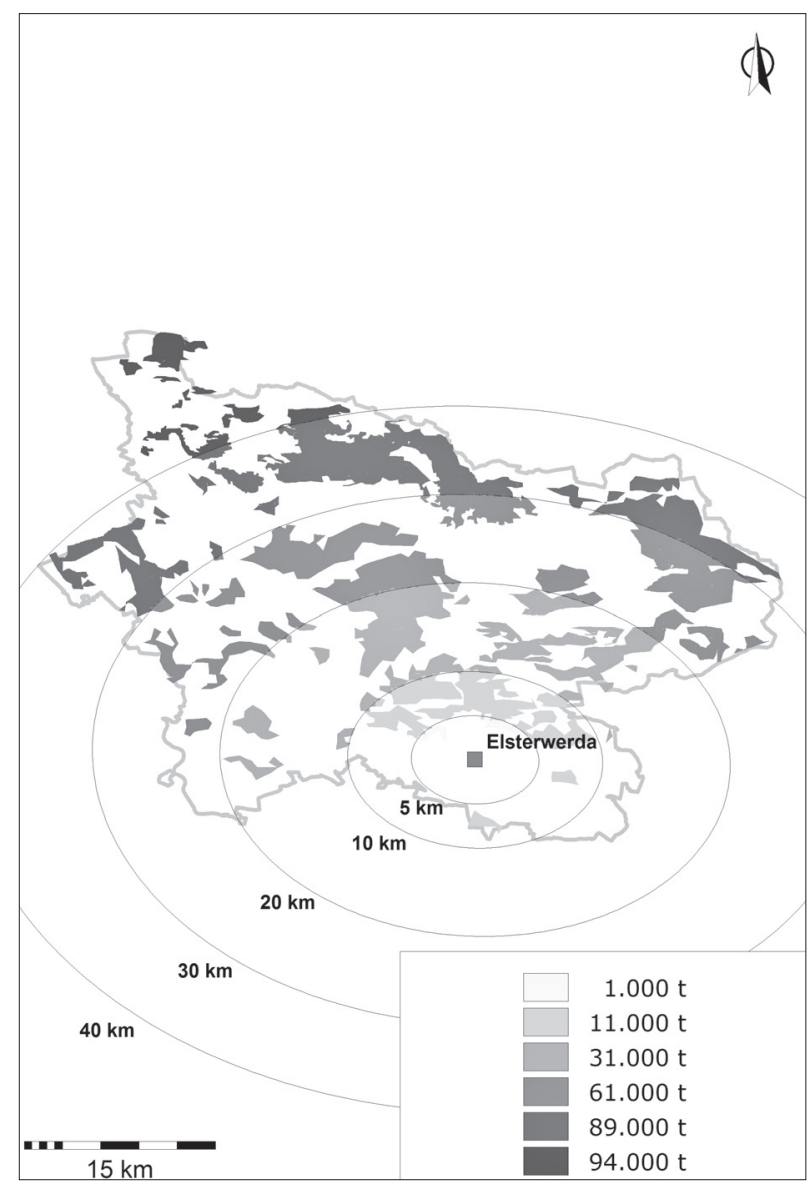

Abb. 5: Räumliche Verteilung der Dendromasse um den Standort Elsterwerda

\section{Ermittlung der Bereitstellungskosten für Waldhackschnitzel}

Für eine Bewertung der Bereitstellungsketten von Waldhackschnitzeln für das Kraftwerk Elsterwerda wird das Blickfeld über die drei Grundprozesse der Logistik - Transport, Umschlag und Lagerung - hinaus erweitert. Die Wahl des Ernteverfahrens und die Aufbereitung der Dendromasse sind bedeutende Kostenfaktoren und beeinflussen die Transport- und Umschlagskosten wesentlich.

Es werden die in Abb. 6 dargestellten exemplarischen Bereitstellungsketten angenommen. Die Aufbereitung der Dendromasse, d. h. die Herstellung von Waldhackschnitzeln, wird in Kombination mit dem Transport durchgeführt. Die optimale Abstimmung der Kapazitäten der einzelnen Teilprozesse führt hierbei zu minimalen Bereitstellungskosten. Die technischen Leistungsvorgaben der Maschinen sind berücksichtigt. Maßgebend für den Hackvorgang sind die Vorgaben durch das Kraftwerk Elsterwerda hinsichtlich der Form und Größe der Hackschnitzel. Zur Vermeidung eines Umschlages werden die Hackschnitzel direkt aus dem Hacker in einen bereitstehenden Anhänger oder Wechselcontainer eingeblasen.

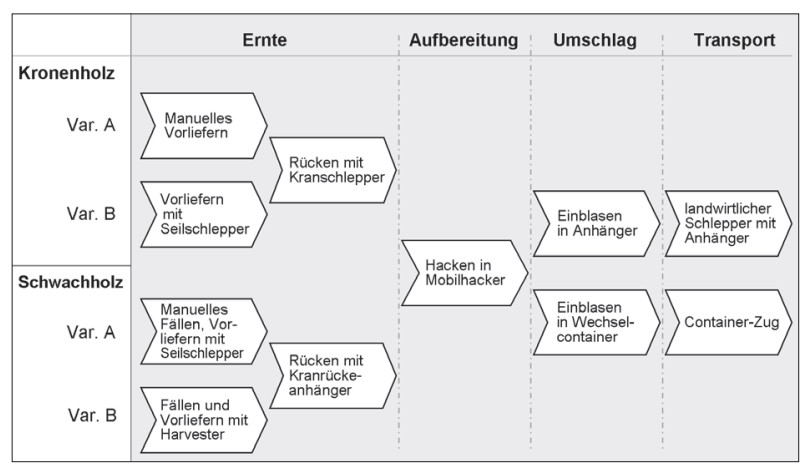

Abb. 6: Exemplarische Bereitstellungsketten für Dendromasse

Die monetäre Bewertung der einzelnen Schritte der Bereitstellung erfolgt auf der Grundlage von Daten des Kuratoriums für Wald- und Forstwirtschaft (KWF 2004) und der Bayrischen Landesanstalt für Wald und Forstwirtschaft (LWF 2003).

Neben den Prozessen der Ernte und Aufbereitung bestimmt die räumliche Verteilung der Dendromassepotentiale um den Standort Elsterwerda die Bereitstellungskosten.

Für die Berechnungen gilt, dass im Umkreis von $5 \mathrm{~km}$ ausschließlich Landwirtschaftliche Züge den Transport der Dendromasse übernehmen, bei längeren Transportentfernungen werden Container-Lkw eingesetzt.

Es wurden Beispielrechnungen für Transporte mit verschiedenen Anhängern durchgeführt. Das Ladevolumen der Anhänger der landwirtschaftlichen Züge wird dabei mit 14,9 m³ (klein) bzw. 18,7 m³ (groß), die der Abrollcontainer mit $35 \mathrm{~m}^{3}$ angenommen. Der Transport mit Abrollcontainern hat sich grundsätzlich mit 2 Anhängern kostengünstiger dargestellt. Auf dieser Grundlage werden alle weiteren Betrachtungen geführt.

Unter Berücksichtigung der relevanten Parameter ergeben sich die in Abb. 7 dargestellten Gesamtkosten der Bereitstellung. 


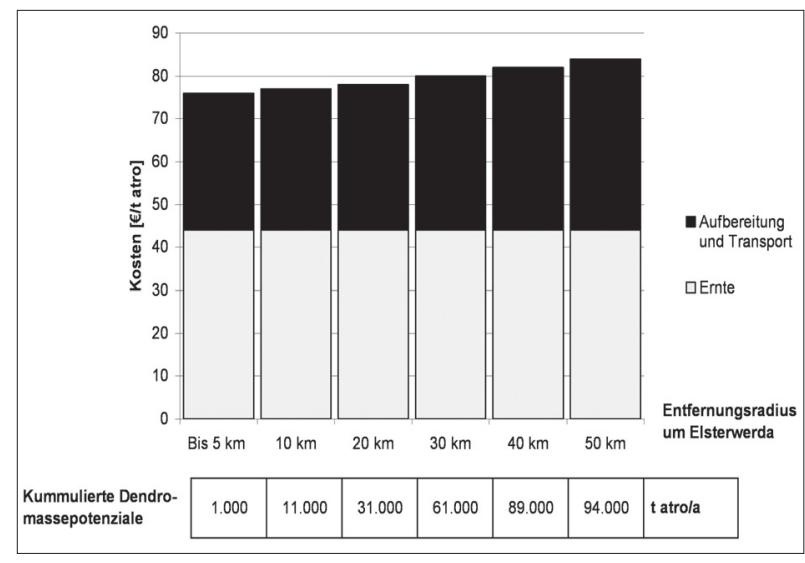

Abb. 7: Bereitstellungskosten und Dendromassepotenziale in Abhängigkeit des Entfernungsradius um den Standort Elsterwerda, Quellen: Leible et al (2003), KWF (2004)

Die ermittelten Bereitstellungskosten von über $80 € / \mathrm{t}$ atro übersteigen den zurzeit für Altholz gezahlten Preis erheblich. Dieser wird, basierend auf Schätzungen nach Angaben von Leible et al (2003) und einer mündlichen Mitteilung des Kraftwerkbetreibers in Elsterwerda mit $25 € / t$ atro angenommen. Das Waldholz ist somit preislich in dieser besonderen Situation nicht konkurrenzfähig.

Grund für die hohen Bereitstellungskosten ist vor allem die bisher mangelnde Effizienz der Prozesskette. Durch den räumlich verteilten Anfall geringer Mengen Dendromasse ist ein häufiges Umsetzen der Ernte- und Aufarbeitungsmaschinen (Hacker) notwendig. Bei unzureichender Bündelung von Maßnahmen, führt dies zu hohen unproduktiven Zeiten und hohen Umsetzkosten. Zudem führt eine ungenaue Abstimmung zwischen anfallender Dendromasse, Dimensionierung des Hackers und Bereitstellung entsprechender Transportfahrzeuge $\mathrm{zu}$ hohen Stillstandzeiten und damit zu hohen Personalund Maschinenkosten. In diesem Bereich bestehen große Optimierungspotenziale.

\section{Bewertung des Versorgungskonzepts}

Mit insgesamt 8 Mio. €/a übersteigen die Kosten für die Bereitstellung von Waldhackschnitzeln deutliche das im Biomassekraftwerk zur Verfügung stehende Budget für die Rohstoffbeschaffung. Vor dem Hintergrund der sich verknappenden Altholzpotentiale und dem erwarteten Preisanstieg erscheint es dennoch sinnvoll, einen möglichen Beitrag von Waldholz zur Versorgung des Standorts abzuschätzen. Dazu werden die finanziellen Auswirkungen einer Beimischung von Waldhackschnitzeln zur bestehenden Versorgung mit Altholz untersucht.

Die Beschaffungskosten ergeben sich aus der Summe der Bereitstellungskosten für Altholz bzw. Waldhackschnitzel. Über den jeweiligen prozentualen Anteil des Sortiments wird ausgehend von den benötigten $96.000 \mathrm{t}$ atro Holz, der jeweilige absolute Versorgungsanteil in $t$ atro pro Jahr errechnet. Durch die monetäre Bewertung des Altholzes mit $25 € / t$ atro und des Waldholzes entfernungsabhängig nach dem Einzugsradius nach Tabelle 5 und der Summierung beider Ergebnisse ergeben sich die Gesamtkosten wie in Abb. 8 dargestellt.

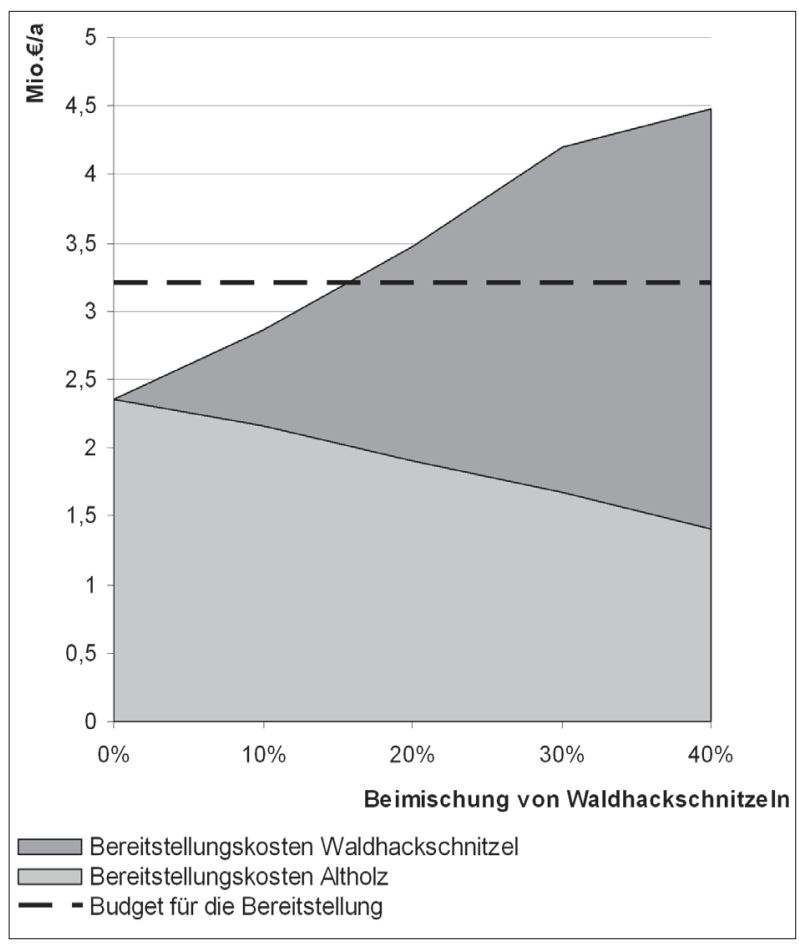

Abb. 8: Bereitstellungskosten in Abhängigkeit des Waldholzanteils an der Versorgung und Vergleich zum verfügbaren Jahresbudget für die Biomassebereitstellung

Aus der Abb. wird deutlich, dass mit einem höheren Anteil Waldhackschnitzel die Gesamtkosten der Bereitstellung stark anwachsen. Bei einer Zumischung von 40 \% Waldhackschnitzel verdoppeln sich die Bereitstellungskosten nahezu gegenüber der reinen Altholzvariante. Dies ist auf die ungleich höheren Bereitstellungskosten des Waldholzes gegenüber dem Altholzpreis zurückzuführen. Die höheren Transportkosten, bedingt durch das größere Einzugsgebiet bei der Waldholzbeschaffung, spielen eine eher untergeordnete Rolle. Die Transportentfernungen sind im regionalen Maßstab so gering, dass die hierfür anfallenden Kosten nur einen Bruchteil derer ausmachen, die für die Ernte und die Aufbereitung der Dendromasse anfallen.

Unbetrachtet bleibt in dieser Abschätzung die Nutzungskonkurrenz durch andere Biomasseanlagen und private Abnehmer. Für eine genauere Ermittlung der verfügbaren Mengen, müssten diese berücksichtigt werden. In der Praxis würde sich die Versorgung höchstwahrscheinlich aus einem größeren Umkreis mit entsprechend höheren Transportkosten gestalten. Da zudem die Kosten der Bereitstellung Schwankungen unterliegen können und dieser Unsicherheit Rechnung getragen werden muss, ist ein 10\%iger Anteil an Waldholz als realistisch einzustufen. Eine kontinuierliche Zumischung ist wichtig, um technische Probleme zu vermeiden.

Unter den derzeitigen Bedingungen, ist die Umstellung dieser auf den Brennstoff Altholz ausgelegten Anlage auf Waldrestholz also nicht empfehlenswert. Es stellt sich daher die Frage, ob eine ökonomisch sinnvolle Nutzung von Dendromasse zum jetzigen Zeitpunkt überhaupt möglich ist, und welche Perspektiven sich zukünftig ergeben.

Deshalb soll im Folgenden untersucht werden, ob ein anderer Anlagentyp am Standort Elsterwerda betriebswirtschaftlich erfolgreich mit Waldhackschnitzeln betrieben werden könnte. Es gibt eine Vielzahl unterschiedlicher 
Anlagen zur Wärme- und Strombereitstellung, die ausschließlich auf die Nutzung von Dendromasse ausgelegt sind. Aufgrund unterschiedlicher technischer Anforderungen im Vergleich zur Anlage in Elsterwerda, liegen die Investitionskosten und somit auch die jährliche Fixkostenbelastung auf einem niedrigeren Niveau. Im Folgenden wird für zwei exemplarische Anlagentypen kleiner und mittlerer Dimension (siehe Tabelle 4) untersucht, welche finanziellen Mittel maximal für die Beschaffung von Dendromasse aufgewendet könnten.

\begin{tabular}{|l|l|l|}
\hline Basisdaten & $\begin{array}{l}\text { Beispiel 1: 500 kW } \\
\text { Kesselanlage }\end{array}$ & $\begin{array}{l}\text { Beispiel 2: 10 MW } \\
\text { Biomasseanlage }\end{array}$ \\
\hline Volllaststunden [h/a] & 2.000 & $2 \times 6.000$ \\
\hline $\begin{array}{l}\text { Erzeugte jährliche Wärme- } \\
\text { menge [MWh/a] }\end{array}$ & 1.000 & 34.200 \\
\hline $\begin{array}{l}\text { Erzeugte jährliche Strom- } \\
\text { menge [MWh/a] }\end{array}$ & - & 9.000 \\
\hline Biomassebedarf [t atro/a] & 330 & $11.500 \mathrm{t}$ \\
\hline Jährliche Fixkosten [€/a] & 36.000 & 2.070 .000 \\
\hline
\end{tabular}

Tab. 4: Technische Daten der Beispielanlagen, Quellen: FNR (2005), Leible et al (2003)

Die maximal möglichen Bereitstellungskosten für diese beiden Beispielanlagen werden aufgrund ihrer Stromund Wärmeproduktion und der jeweiligen Fixkostenbelastung sowie in Abhängigkeit vom Ölpreis berechnet (siehe Abb. 9). Die ermittelten Werte werden zu den errechneten Bereitstellungskosten am Standort Elsterwerda in Relation gesetzt.

$$
\rho^{\prime}
$$

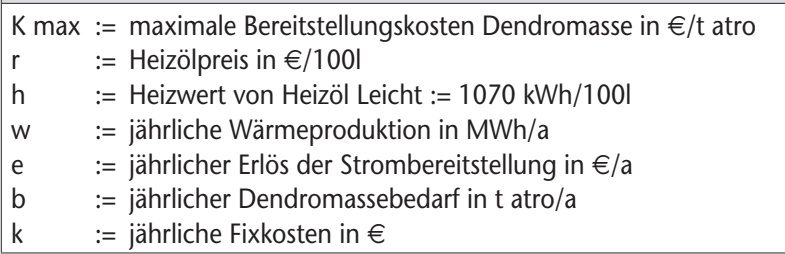

Abb. 9: Formel zur Berechnung der maximal möglichen Bereitstellungskosten Dendromasse für ein Biomasseheiz(kraft)werk

Der Ertrag der Heizanlage ist nach dem aktuellen Preis für Heizöl zu bewerten. Im April 2006 stieg dieser zum dritten Mal innerhalb des letzten Jahres auf über $65 € / 1001$ an - Tendenz weiter steigend (Tecson, 2006). Bei diesem Heizölpreisniveau könnten Bereitstellungskosten für die Dendromasse von $75 € / t$ atro (Beispielanlage 1 ) bzw. $86 € /$ t atro (Beispielanlage 2) in Kauf genommen werden. Am Standort Elsterwerda, liegen nach den vorliegenden Berechnungen die Bereitstellungskosten im Umkreis von $5 \mathrm{~km}$ bei $76 € / \mathrm{t}$ atro, innerhalb von $10 \mathrm{~km}$ bei $80 € / \mathrm{t}$ atro. Beide Beispielanlagen könnten hier also betriebswirtschaftlich erfolgreich auf der Basis von Waldhackschnitzeln betrieben werden.

Aus heutiger Sicht ist also das wirtschaftliche Betreiben von Biomasseheiz(kraft)werken mit Waldrestholz möglich. Vor dem Hintergrund, der erwarteten Preissteigerung bei den fossilen Energieträgern, wird sich die Situation in Zukunft noch stärker zugunsten der Dendromasse verschieben. Abb. 10 zeigt, dass bei steigendem Ölpreis auch wesentlich höhere Bereitstellungskosten für die Dendromasse in Kauf genommen werden könnten.

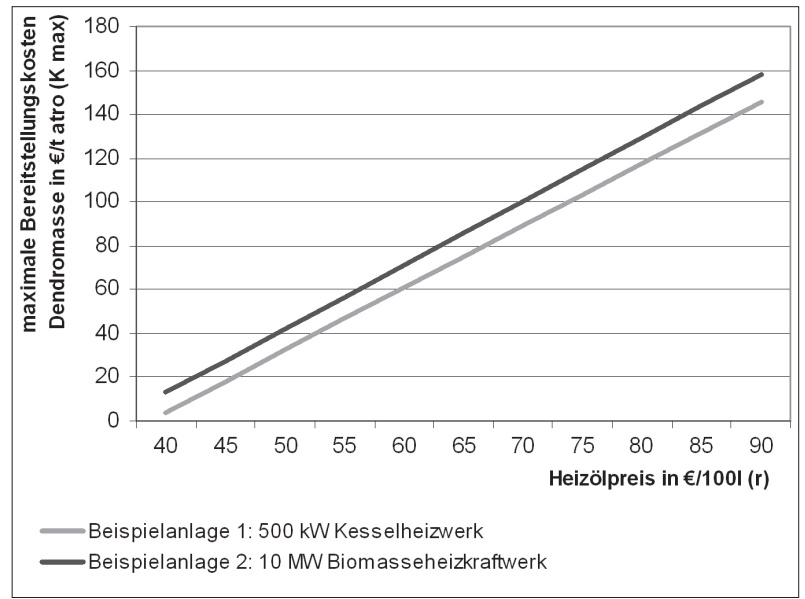

Abb. 10: Maximal mögliche Bereitstellungskosten Dendromasse (K max) für die beiden Beispielanlagen in Abhängigkeit des Ölpreises ( $r$ ).

\section{Fazit und Empfehlungen}

Unter den derzeitigen Bedingungen ist eine Versorgung des Biomassekraftwerks Elsterwerda ausschließlich mit Waldholz nicht möglich. Gründe sind die zurzeit hohe preisliche Differenz zwischen Wald- und Altholz sowie die technische Auslegung der Anlage auf Altholz. Vor dem Hintergrund der Entwicklung auf dem Altholzmarkt ist hingegen eine Zufeuerung von Waldholz in bestimmten Maßen zu empfehlen. Sie erhöht die Flexibilität auf dem Beschaffungsmarkt und eröffnet Perspektiven für eine zukünftige Umstellung der Anlage. Unter den derzeitigen Bedingungen wird eine Zumischung von etwa 10\% empfohlen. Bei einer Senkung der Bereitstellungskosten für Waldrestholz oder bei steigenden Altholzpreisen sind höhere Anteile möglich.

Das Ziel, mehr Strom und Wärme aus regionaler Biomasse bereitzustellen, ist jedoch grundsätzlich nicht in Frage zu stellen. Derzeit lohnt sich vor allem die Investition in kleinere Heiz(kraft)werke auf kommunaler Ebene, die technisch für die Nutzung von frischer Dendromasse ausgelegt sind. Anhand zweier Beispiele wurde gezeigt, dass solche Anlagen bereits heute kostendeckend mit einer ausschließlichen Versorgung durch Waldrestholz betrieben werden können. Bei anhaltend steigendem Ölpreis verschieben sich die Bedingungen weiter zugunsten der Dendromasse.

Die Energiebereitstellung wird am effizientesten in kleinen unabhängigen Kreisläufen geregelt. Der Koordinationsaufwand ist hier relativ gering und kleinere Versorgungsnetzwerke können grundsätzlich auch flexibler auf Änderungen am Markt reagieren. Transportentfernungen bis zu $50 \mathrm{~km}$ haben geringen Einfluss auf die Gesamtkosten, gewinnen bei höheren Entfernungen aber an Bedeutung.

Um langfristig in mittleren bis großen Kraftwerken mit dem Einsatz von Dendromasse zur Energiegewinnung planen zu können, muss die Effizienz der Bereitstellungs- 
kette gesteigert werden. Es ist vor allem bei den Prozessen der Ernte und Aufbereitung anzusetzen. Dies ist zum einen durch technische Neuerungen möglich, zum anderen können organisatorische Maßnahmen Abhilfe schaffen.

Eine reibungslose Kommunikation zwischen den Akteuren und eine effektive Koordination der Abläufe ist die Voraussetzung für den effizienten Einsatz von Personal und Maschinen. Vor allem die Prozesse der Aufbereitung und des Transportes bieten Optimierungspotenziale. Der Anfall von Dendromasse ist zudem in den meisten Fällen sehr kleinvolumig. Das größte ungenutzte Potential ist im Kleinprivatwald zu finden. Hier ist es essentiell, Maßnahmen regional zu koordinieren. Forstbetriebsgemeinschaften können dabei eine Schlüsselrolle spielen.

Technische Innovationen und die regionale Bündelung und Koordinierung von Ressourcen bilden damit die Grundlage für den zukünftigen Erfolg der Energiegewinnung aus Dendromasse.

\section{Literatur}

Bilke, G. (2005): Forstliche Dendromassepotentiale im Landkreis Elbe-Elster, schriftliche Mitteilung, Landesforstanstalt Eberswalde

BMU (2002): Verordnung über Anforderungen an die Verwertung und Beseitigung von Altholz, Bundesministerium für Umwelt, Naturschutz und Reaktorsicherheit, Berlin

BMU (2005): Erneuerbare Energien in Zahlen - Nationale und internationale Entwicklung, Stand Dezember 2005, InternetUpdate, Bundesministerium für Umwelt, Naturschutz und Reaktorsicherheit, Berlin

BMU (2006): Wirtschaftfaktor Umwelt - Innovation, Wachstum und Beschäftigung durch Umweltschutz, Bundesministerium für Umwelt, Naturschutz und Reaktorsicherheit, Berlin

BMWI, BMU (2006): Energieversorgung für Deutschland - Statusbericht für den Energiegipfel am 3. April 2006, Berlin

Dieter, M.; Englert, H. (2001): Abschätzung des Rohholzpotentials für die energetische Nutzung in der Bundesrepublik Deutschland, Bundesforschungsanstalt für Forst- und Holzwirtschaft, Arbeitsbericht des Instituts für Ökonomie 2001/1, Universität Hamburg, Hamburg

EEG (2004): Gesetz für den Vorrang Erneuerbarer Energien

FNR (2005): Leitfaden Bioenergie - Planung, Betrieb und Wirtschaftlichkeit von Bioenergieanlagen, Fachagentur Nachwachsende Rohstoffe, Gülzow

Kaltschmitt, M.; Hartmann, H. (Hrsg.) (2001): Energie aus Biomasse - Grundlagen, Techniken und Verfahren, Springer, Berlin u. a.

KWF 2004: Holzernteverfahren - Vergleichende Erhebung und Beurteilung der Holzernteverfahren in der Bundesrepublik Deutschland, KWF Bericht Nr. 25, CD-ROM, Kuratorium für Wald- und Forstwirtschaft, Groß Umstadt

LDSB (2003): „Bodennutzung der landwirtschaftlichen Betriebe im Land Brandenburg", Statistische Berichte C I 1 - j / 03, Landesbetrieb für Datenverarbeitung und Statistik Brandenburg

Leible, L., et al (2003): Energie aus biogenen Rest- und Abfallstoffen -bereitstellung und energetische Nutzung organischer Rest- und Abfallstoffe sowie Nebenprodukte als Einkommensalternative für Land- und Forstwirtschaft - Möglichkeiten,
Chance und Ziele, Forschungszentrum Karlsruhe in der Helmholz-Gemeinschaft, Wissenschaftliche Berichte FZKA 6882, Forschungszentrum Karlruhe $\mathrm{GmbH}$, Karlsruhe

Tecson (2006): Entwicklung des Heizölpreises 2003-Tagesaktuell, http://tecson.de/pheizoel.htm, 12.05.2006

Weimar, H.; Mantau, U. (2005): Kapazitäten der Biomasse(heiz) kraftwerke und ihre Rohstoffversorgung in Deutschland in: BBE - Bundesverband BioEnergie e.V., 2005; HolzEnergie 2005 - Internationaler Fachkongress für Holzenergie, Tagungsband, Bonn

Wittkopf, S.; Hömer, U.; Feller, S. (2003): Bereitstellungsverfahren für Waldhackschnitzel - Leistungen, Kosten, Rahmenbedingungen, LWF Wissen Nr. 38, Bayerische Landesanstalt für Wald und Forstwirtschaft

\section{Autoren}

Dipl.-Wirtsch.-Ing. (FH) Paul Fiedler

Absolvent der Technischen Fachhochschule Wildau paulfiedler@gmx.de

\section{Prof. Dr.-Ing. Herbert Sonntag}

Technische Fachhochschule Wildau

Fachbereich Ingenieurswesen/Wirtschaftingenieurwesen - Verkehrslogistik

Tel. +49 3375 508-511, -924

hsonntag@igw.tfh-wildau.de

\section{Dipl.-Forstw. Mareike Schultze}

Technische Fachhochschule Wildau

Fachbereich Ingenieurswesen/Wirtschaftingenieurwesen - Verkehrslogistik

Tel. +493375 508-511

schultze@igw.tfh-wildau.de 